\section{Importance of differentiating Mycobaterium bovis in tuberculous meningitis}

\author{
Alejandra González-Duarte, 1 \\ Alfredo Ponce de León, 2 \\ José Sifuentes Osornio² \\ 1Department of Neurology and \\ 2Microbiology of the Instituto Nacional \\ de Ciencias Médicas y Nutrición Salvador \\ Zubiran, México City, Mexico
}

\section{Abstract}

The aim of the article is to describe the principal findings among patients with M.tuberculosis and $M$. bovis CNS infection. Mycobacterium tuberculosis is one of the most common infectious agents that cause death and neurological sequelae around the world. Most of the complications of CNS TB can be attributed to a delay in the diagnosis. Unfortunately, there are no specific diagnostic tools to support an early diagnosis. Other prognostic factors different from delay in treatment have not been identified. Clinical, radiological and laboratory characteristics were analyzed retrospectively from the medical files of all the patients admitted with the diagnoses of tuberculosis. Of 215 patients admitted with systemic tuberculosis, 64 (30\%) had a neurological infection. Positive cultures were found in 54 (84\%) cases, 18 (33\%) in the CSF and the rest in other fluids or tissues. Adenosin deaminase (ADA) enzyme determination was more sensitive than $M$. tuberculosis PCR in the CSF for supporting an early diagnosis. In addition to a later clinical stage and treatment lag, positive CSF cultures $(\mathrm{P}=0.001)$ and the presence of $M$. bovis $(\mathrm{P}=0.020)$ were prognostic factors for a worse outcome. Neither older age, the presence of tuberculomas versus meningeal enhancement, or HIV co-infection, was associated to a worse prognosis. The isolation of $M$. bovis subspecies was more common that previously reported, and it was associated to the development of parenchymal lesions $(\mathrm{P}=0.032)$ when compared to M. tuberculosis. In this study, positive CSF cultures for $M$. tuberculosis and further identifying $M$. bovis species were additional prognostic factors for worse outcome. Positive cultures in systemic fluids other than CSF, even when the patient had no obvious systemic manifestations, and ADA determination in the CSF were noteworthy diagnostic tools for the diagnosis.

\section{Introduction}

Mycobacterium tuberculosis is one of the most common infectious agents that cause death and neurological sequelae around the world. Only in Mexico, there are 40,000 cases of active tuberculosis, and around 2500 cases are multi-drug resistant. ${ }^{1}$ It is estimated that the central nervous system (CNS) is involved in $5-10 \%$ of the extra-pulmonary cases, accounting for $1.3 \%$ of the total number of cases. ${ }^{2}$ The diagnosis of CNS tuberculosis is challenging, mostly because the initial symptoms may be identical to bacterial or viral meningitis. As a result, most of the patients receive multiple incorrect treatments, which may improve the symptoms temporary, but lengthens the time for diagnosis and worsens the prognosis.

Most of the complications of CNS tuberculosis can be attributed to the direct result of a delay in the diagnosis, with an estimated 3.7fold risk for a fatal outcome when the treatment is begun after 5 days of the initiation of symptoms. ${ }^{3}$ Unfortunately, there are no specific diagnostic tools to support an early diagnosis. Isolating M. tuberculosis from CSF cultures is the only definite diagnostic method, but it has a very low sensitivity and becomes positive up to 6 to 8 weeks later. Other ancillary tests for the diagnosis of CNS tuberculosis are less specific, and most have been extrapolated for the diagnosis of pulmonary tuberculosis. ${ }^{4}$ In this study we aimed to identify the most common clinical, radiographic, and laboratory findings in patients with tuberculosis and CNS involvement in the Mexican population, and we compared them to similar series from the literature.

\section{Materials and Methods}

We reviewed retrospectively the medical files of all patients with the diagnosis of pulmonary or extra-pulmonary tuberculosis, CNS tuberuculosis and tuberculous meningitis admitted to a tertiary-care level hospital in Mexico City from 1999 to 2009. Diagnosis of CNS tuberculosis was done according to international recognized clinical, radiological and laboratory criteria. ${ }^{5}$ Clinical criteria included fever, headache, and nucal rigidity. Laboratory criteria included CSF pleocytosis greater than 10 cells per $\mathrm{mm}^{3}$ or proteins above $30 \mathrm{mg}$ per deciliter with negative bacterial or fungal CSF cultures. Radiological criteria included hydrocephalus, tuberculomas, cerebral infarcts, meningeal enhancement, or exudates. In addition, the results of the following tests were recollected: i) presence of mycobacteria in the CSF or cerebral tissue; ii) polymerase chain
Correspondence: Alejandra González-Duarte, Neurology Department, Insituto Nacional de Ciencias Médicas y Nutrición Salvador Zubirán. Vasco de Quiroga 15, Sección XVI, Tlalpan, México D.F.

Tel: +52.54851697 - Fax: +52.54851328 .

E-mail: gonzalezduarte@aol.com

Key words: CNS tuberculosis, tuberculous meningitis, M. tuberculosis, M. bovis.

Acknowledgment: the authors would like to thank to all the personal of the microbiology laboratory and especially to Miriam Bobadilla because without their work this manuscript would have not been possible.

Contributions: the authors contributed equally.

Conflict of interest: the authors report no conflicts of interest.

Funding: No funding was received for the elaboration of this manuscript.

Received for publication: 25 may 2011.

Revision received: 8 July 2011.

Accepted for publication: 30 Augudt 2011.

This work is licensed under a Creative Commons Attribution NonCommercial 3.0 License (CC BYNC 3.0).

CC Copyright G. Shishu et al., 2011

Licensee PAGEPress, Italy

Neurology International 2011; 3:e9

doi:10.4081/ni.2011.e9

reaction (PCR) tests for $M$. tuberculosis in the CSF; iii) microscopic examination of the CSF; iv) cultures or staining of other corporal fluids such as gastric aspirate; urine, feces, pleuritic fluid or ascitis; v) cultures or stain of non-cerebral tissues; vi) tuberculin skin test (PPD).

A definite diagnosis of meningeal tuberculosis was done when a positive culture or staining for M. tuberculosis was present in the CSF or cerebral tissue. A probable diagnosis was done when the clinical picture was compatible, in addition to positive cultures or stains in other non-CNS fluids or tissues. A possible diagnosis was performed when there was a suggestive clinical picture, history of systemic tuberculosis, and improvement with antituberculosis treatment.5-6

CSF and other corporal fluids and tissues were processed in all the patients with a clinical suspicion of tuberculosis according to the clinical criteria and the decision of the physician in charge. All clinical specimens were cultured in enrichment medias for aerobic and anaerobic bacteria, mycobacteria and fungus. After the Ziehl-Neelsen stain, all the samples were cultured in solid media (LöwensteinJensen modified with asparagine) (DIFCO, Mex), and in liquid media Bactec 12B or MGIT 
(Becton, Dickinson, Cockeysvill MD, USA), according to the manufacturer instructions. The cultures were identified by DNA samples (Geneprobe, San Diego, CA, USA) or with the traditional biochemical methods. The drug susceptibility essays to first line ATB drugs were done through the radiometric method Bactec 460 TB (Becton Dickinson). The PCR was nested with the following oligonucleic acids of the insertion sequence IS6110; the external oligonucleids G015'CGGGACCACCCGCGGCAAAGCCCGCAGGAC 3 'and G02 5'CATCGTGGAAGCGACCCGCCAGCCCAGGAT 3 produced a fragment of $219 \mathrm{pb}$; the internal oligonucleids Tb4 5'CCTGCGAGCGTAGGCGTCGG 3' and Tb5 5'CTCGTCCAGCGCCGCTTCGG $3^{\prime}$ that produced a fragment of $123 \mathrm{pb}$.

Clinical manifestations were classified according to the British Medical Research Council Criteria ${ }^{3,5,6}$ in three stages: Stage I (early): non-specific symptoms, the patient was conscious and without neurological signs; Stage II (intermediate): there was altered mental status but not comatose, or there were focal neurological signs such as cranial nerve involvement or hemiparesis; Stage III (advanced): patients were lethargic or comatose or had severe neurologic alterations such as seizures or involuntary movements.

After initiating anti-tuberculosis treatment and steroids, we registered all new neurological complications including the new formation or enlargement of cerebral lesions, development of hydrocephalus, new onset of seizures or cranial nerve paralysis, occurrence of intracranial hypertension, coma, or death. Clinical outcome at the end of the hospitalization period was registered using the modified Rankin Scale where 1 stands for the absence of neurologic complications, 2 for the presence of non-incapacitating neurologic sequelae, 3 for the presence of incapacitating neurologic sequelae, and 4 for death.

All of the clinical, laboratory and radiological characteristics were analyzed through univariate and multivariate logistic regression in patients with good outcome and bad outcome, using as final parameters death, non-incapacitating, and incapacitating neurologic sequelae. The Fisher's Exact test was used for the categorical analysis and the Wilcoxon Test for the non-parametric analysis. SPSS version 16 for Mac was used for the statistical analysis. A value of $P=0.05$ or less was used as statistically significant. This retrospective study was approved by the IRB ethical standards.

\section{Results}

\section{Epidemiological characteristics}

From 1999 to 2009, 215 patients were diagnosed with systemic tuberculosis; of them, 64
(30\%) had clinical manifestations of CNS involvement and were included in the study. Fifty patients (78\%) were men. Mean age at onset was 45 years old (range 21-83). Fourteen patients (22\%) were HIV positive. Other comorbidities varied widely. Only four (6\%) patients came from the north states, including two from the United States, and two from the south states. The rest came from the central states of the country. Diagnosis was definite in 44 patients (68.8\%), probable in 14 (21.9\%), and possible in 6 (9.4\%).

\section{Characteristics at entry}

Of the 64 patients with CNS tuberculosis, the neurologic symptoms were the only manifestation of the disease in $44 \%$ of the patients, $38 \%$ had neurologic and systemic manifestations at onset, and only $18 \%$ had neurological involvement after more than one month of the diagnosis of systemic tuberculosis. The mean interval of time between the initial clinical manifestations, and hospital admission was 2 months $( \pm 6 ; 0$-36 days).

\section{Clinical course}

Forty patients (62\%) had meningitis or menigoencephalitis as the initial presentation, 20 (31\%) had focal neurological signs suggestive of tuberculomas or stroke, and the rest had a combination of both presentations. Sixty-six percent of the patients had a Glasgow Coma Score (GCS) below 15 points and one third of the patients had meningeal signs. Nineteen patients (30\%) had seizures at onset and 9 (14\%) had stroke. According to the MRC criteria, 11 patients (17\%) were in Stage I, 26 (49\%) in Stage II and 27 (43\%) in Stage III.

\section{Laboratory findings}

Intradermal purified protein (PPD) reaction was assessed in 27 patients and had a positive value of 15 to $80 \mathrm{~mm}$ in 7 (25\%). Ziehl-Neelsen stains were positive in 8 cases, three in the CSF, two in stools, and the rest in miscellaneous tissues (sputum, ascites, lymph nodes, and cerebral tissue). Positive cultures were found in 54 (84\%) cases, 18 in the CSF, 14 in the pulmonary biopsies, and the rest in other tissues or fluids (lymph nodes, cerebral biopsy, peritoneal fluid, urine, eye, bone marrow and bone biopsies). Forty-six cultures were positive for M. tuberculosis and 8 cultures were further identified as $M$. bovis. Twenty-five (39\%) patients had hyponatremia $(\mathrm{Na}<130 \mathrm{mEq})$ at entry, and 4 (6.25\%) developed it later (SIADH).

\section{Radiographic findings}

All the patients had an initial brain image performed; three patients had a CT scan, 10 patients had an MRI, and 51 patients had CT scan and MRI. Initial findings were parenchy- mal lesions (tuberculomas) in 21(32\%), meningeal enhancement in 13 (20\%), hydrocephalus in 6 (9.4\%), and stroke in 9 (14\%). Follow-up MRI was performed in 28 patients, on those, new findings were seen in $20(83 \%)$ patients; further development of stroke was seen in 6 (21.4\%), of hydrocephalus in 6 (21.4\%), of new parenchymal lesions in 5 (17.8\%), and of pachymeningitis in 3 (10.7\%). There was improvement in 4 cases and similar findings as the initial image in the rest. No spinal cord image was performed in our patients in search of arachnoiditis or intraspinal tuberculomas.

\section{Lumbar puncture}

All the patients had at least one LP. Initial CSF analysis was abnormal in 55 (85\%) patients. Subsequent CSF analysis was done in 33 patients, with a time interval between one and 270 days. The results are shown in Table 1. CSF PCR for M. tuberculosis was positive in 22 (55\%) of 40 patients in which the test was performed. Of the 26 patients with adenosindeaminse (ADA) enzyme determination in the CSF, 25 (96\%) had a positive result. ADA concentrations varied between 1 and 50 UI, with a mean and median of $8 \mathrm{UI}$.

\section{Treatment}

All the patients were hospitalized. The time interval between the hospital admission and the initiation of treatment was 21 days (R:0-5540 , median 9). Thirty-seven patients (57.5\%) received empirical treatment with the combination of a third-generation cephalosporin and vancomycin for suspicion of bacterial meningitis. Twenty-nine patients (45\%) had acyclovir or amphotericin $\mathrm{B}$ added to the previous regimen before initiating anti-tuberculosis treatment. Fifty patients (78\%) received steroids before the anti-tuberculosis (ATb) treatment for the suspicion of bacterial meningitis or because there were already prescribed for other reasons. All the patients were initiated with four ATb medications (rifampicin, isoniazid, pyrazinamide and ethambutol) for at least two months before receiving the maintenance phase. Mean time of ATb therapy was $11.9 \pm 7$ months. Fourteen patients (21\%) required a ventriculo-peritoneal shunt to treat hydrocephalus.

\section{Prognostic factors and outcome}

Forty-five patients (71.4\%) had a bad outcome (Rankin 2, 3 and 4). Twenty-two patients (34\%) suffered permanent neurologic sequelae and 23 (35\%) died. Twenty-five patients (39\%) had neurological deterioration during the hospitalization between the third and 130th days (median 30 days). Thirteen (20\%) patients had new or enlarged cerebral lesions during treatment. Ten patients (15\%) devel- 
oped seizures. Hydrocephalus occurred in 12 (19\%), and stroke in 10 (15.6\%). Of patients with a bad outcome, 12 (18.8\%) were not receiving steroids at the time, steroids were being tapered-down in one, and the rest had them at optimal doses. Three patients with new parenchymal lesions were receiving steroids and ATb treatment at the time of the second MRI, despite steroids, an immune reconstitution syndrome was suspected. The correlation between clinical the characteristics and outcome is described in Table 2.

Univariate analysis showed a statistically significant relation between neurological sequelae and a positive CSF culture $(\mathrm{P}=0.001)$. There was a tendency in the relation between the neurological sequelae and a time interval longer than 10 days between symptoms and treatment $(\mathrm{P}=0.087)$, neurological sequelae and clinical stage II $(\mathrm{P}=0.092)$ and clinical stage III $(\mathrm{P}=0.071)$ at the time of initiating treatment. Notably, neither age above 50 years old, meningeal or cerebral presentation at onset, Glasgow Coma Scale (GCS) at onset, or HIV co-infection were associated with the development of neurological sequelae. Death was significantly associated with a treatment lag of more than 10 days $(\mathrm{P}=0.002)$, and there was a tendency between death and clinical stage III at the time of initiating treatment $(\mathrm{P}=0.051)$ and between death and HIV coinfection $(\mathrm{P}=0.077)$. Multivariate analysis showed that a GCS at entry below 10 points $(\mathrm{P}=0.001)$, and the clinical stage III at entry $(\mathrm{P}=0.001)$, were significantly associated with death. The isolation of $M$. bovis was significantly associated with cerebral lesions $(\mathrm{P}=0.032)$, and neurological sequelae $(\mathrm{P}=0.020)$ in the univariate and multivariate analysis.

\section{Discussion}

Central nervous system involvement was found in $30 \%$ of the patients with M. tuberculosis infection, considerably higher than the 5\% to $10 \%$ observed in other series. ${ }^{2}$ Table 2 compares the most relevant characteristics between this series and other similar series. As shown before, worse outcomes were related to the later clinical presentation and the delay in initiating treatment. The most common factor for delaying the treatment was the suspicion of bacterial meningitis. Other studies ${ }^{3}$ have also observed that the medical delay is usually associated with the use of empiric antibiotics.

Previous studies have already pointed out the value of adenosine deaminse (ADA) enzyme determination in the CSF for the diagnosis of Mtb ${ }^{7,8}$ When a cutoff value of $10 \mathrm{UI}$ was used, ADA showed a sensitivity of $92.7 \%$ and specificity of $97 \%$ for the early diagnosis of CNS tuberculosis. ${ }^{8}$ In this study, ADA determination persisted as a valuable tool for supporting the diagnosis. Moreover, the median value of ADA in this study was $8 \mathrm{UI}$, which is consistent with other studies. ${ }^{7}$ On the other hand, the effectiveness of the polymerase chain reaction (PCR) for CNS tuberculosis continues to be highly controversial. Although sensitivity is considered around $45 \%$ and specificity is $92 \%,{ }^{9}$

previous studies in Mexico showed a much higher a sensitivity of $75 \%$ with a specificity of $59 \% .{ }^{9}$ In this study, PCR was positive only in 22 (55\%) of 40 patients. Our findings supports those of Rana et al. ${ }^{8}$ who reports that CSF ADA determination is a more sensitive indicator than PCR for the diagnosis of CNS tuberculosis. ${ }^{8}$ CSF culture was positive in $18(28 \%)$ and only $3(4.6 \%)$ of the bacillus was found by CSF staining. However, when other tissues and flu-

Table 1. Main characteristics at onset and follow-up between patients.

\begin{tabular}{lcc} 
& Onset & Follow-ulp \\
Clinical findings & $(\mathrm{n}=64)$ & Not relevant \\
CGS $<15$ points & $43(66 \%)$ & \\
Aphasia & $4(6 \%)$ & \\
Cranial nerve alterations & $24(37 \%)$ & \\
Motor weakness & $27(42 \%)$ & \\
Cerebellar signs & $8(12 \%)$ & \\
Meningeal signs & $19(30 \%)$ & $10(15 \%)$ \\
Seizures & $19(30 \%)$ & $31(48 \%)$ \\
Stroke & $9(14 \%)$ & $(\mathrm{n}=28)$ \\
Severe neurological deterioration & $\mathrm{NA}$ & $6(21.4 \%)$ \\
Radiological findings & $(\mathrm{n}=49)$ & $3(10.7 \%)$ \\
Hydrocephalus & $6(9.4 \%)$ & $5(17.8 \%)$ \\
Meningeal enhancement & $13(20.3 \%)$ & $6(21.4 \%)$ \\
Parenquimatous lesions & $21(32.8 \%)$ & $8(28.5 \%)$ \\
Stroke & $9(14.1 \%)$ & $(\mathrm{n}=32)$ \\
Same or improvement & & $550 \mathrm{mg} / \mathrm{dL}$ \\
\hline Lumbar puncture & $(\mathrm{n}=55)$ & \\
Proteins (mg/dL) & $172 \mathrm{mg} / \mathrm{dL}$ & $48 \mathrm{mg} / \mathrm{dL}$ \\
(Mean) & & $90 \mathrm{cel} / \mathrm{mm}^{3}$ \\
Glucose (mg/dL) & $39 \mathrm{mg} / \mathrm{dL}$ & \\
Cells (cells/mm 3 ) & $102 \mathrm{cel} / \mathrm{mm}^{3}$ & \\
(Mean) & & \\
SD, standard deviation. & & \\
\hline
\end{tabular}

Table 2. Comparison of the principal characteristics between similar series.

\begin{tabular}{|c|c|c|c|c|c|}
\hline Variable & $\begin{array}{l}\text { Sutlas } \\
\text { (Turquia) } \\
(\mathrm{n}=61)\end{array}$ & $\begin{array}{l}\text { Misra } \\
\text { (India) } \\
(n=54)\end{array}$ & $\begin{array}{c}\text { Series } \\
\text { Saitoh } \\
\text { (California) } \\
(\mathrm{n}=20)\end{array}$ & $\begin{array}{l}\text { Sheu } \\
\text { ('Taiwán) } \\
\text { (n=105) }\end{array}$ & $\begin{array}{l}\text { México } \\
(n=64)\end{array}$ \\
\hline \multicolumn{6}{|l|}{ Clinical stage } \\
\hline I & 0 & $9(16.6 \%)$ & $4(20 \%)$ & $37(35.2 \%)$ & $11(17.2 \%)$ \\
\hline II & $44 \%$ & $12(22-2 \%)$ & $6(30 \%)$ & $68(64.8 \%)$ & $26(40.6 \%)$ \\
\hline III & $56 \%$ & $33(61.1 \%)$ & $10(50 \%)$ & 0 & $27(42.2 \%)$ \\
\hline $\begin{array}{l}\text { Interval } \approx 0 \text { nset } \\
\text { to admission (mean) }\end{array}$ & $\begin{array}{l}2 \mathrm{~d}-5 \mathrm{~m} \\
\text { (29 days) }\end{array}$ & NA & $\begin{array}{l}8-14 d(45 \%) \\
>15 d(30 \%)\end{array}$ & $\begin{array}{l}1-120 \mathrm{~d} \\
\text { (10 days) }\end{array}$ & $\begin{array}{c}0-60 \\
\text { (30 days) }\end{array}$ \\
\hline $\begin{array}{l}\text { Interval ₹admission } \\
\text { to Atb (mean) }\end{array}$ & NA & NA & $\begin{array}{l}1-8 \\
\text { (1 day) }\end{array}$ & $\begin{array}{c}0-81 \\
(5 \text { days })\end{array}$ & $\begin{array}{c}0-540 \\
\text { (21 days) }\end{array}$ \\
\hline Hidrocephalus & $14(23 \%)$ & $29(53.7 \%)$ & $13(68 \%)$ & $37(35.2 \%)$ & $12(18.7 \%)$ \\
\hline Tuberculomas & $21(34 \%)$ & $12(22.2 \%)$ & $11(58 \%)$ & $7(6.6 \%)$ & $26(40.6 \%)$ \\
\hline Isquemic Stroke & $13(21 \%)$ & $25(46.2 \%)$ & & $29(27 \%)$ & $19(29 \%)$ \\
\hline Neurological sequelae & $19(31 \%)$ & NA & $7(35 \%)$ & $69(65.7 \%)$ & $24(37 \%)$ \\
\hline Seizures & $10(16 \%)$ & $17(31.8 \%)$ & $3(15 \%)$ & NA & $29(45.3 \%)$ \\
\hline Death & $17(27.8 \%)$ & NA & $1(5 \%)$ & $15(14.3 \%)$ & $23(35 \%)$ \\
\hline $\begin{array}{l}\text { Prognostic factors } \\
\text { for worse outcome }\end{array}$ & Stage II-III & $\begin{array}{c}\text { Focal wk } \\
\text { GCS }<6 \\
\text { SSEP }\end{array}$ & None & $\begin{array}{c}\text { OT > }>\text { d } \\
\text { Stage prg. }\end{array}$ & $\begin{array}{l}\text { OT }>10 \mathrm{~d} \\
\text { HIV }+ \\
\text { M. bovis } \\
\text { Stage III }\end{array}$ \\
\hline
\end{tabular}

GCS, Glasgow Coma Scale; SSEP, Somatosensory evoked potentials; OT, onset of treatment; WK, weakness; PGR, progression. 
ids were tested, isolation of the mycobacterium increased to $84 \%$ by culture and $12.5 \%$ by staining, even when the patient had no obvious systemic manifestations.

Of particular relevance was the fact that $15 \%$ of the CSF cultures showed $M$. bovis growth. The worldwide frequency, pathogenesis or mode of transmission of the bovine agent infection in humans is not known. It is possible that despite strict sanitary measures, its frequency is higher than estimated, especially in developing countries where there is a lack of strict control in livestock. ${ }^{10,11}$ Notably, none of the Asian studies mentioned the presence of $M$. bovis. However, Saitoh et al. ${ }^{6}$ showed 3 out of 20 of their patients had positive cultures for $M$. bovis. It is worth mentioning that in their study almost all the patients had a Hispanic ancestry, and most lived in Mexico or in the border between Mexico and the United States. While the mode of transmission is uncertain, some of our patients recognized the ingestion of raw milk. Reactivation from previous childhood exposure and human to human transmission are possible, but the absence of extrapulmonary disease, the absence of contact between affected cases and the poor differentiation of the genotypes suggest that the most common cause of transmission in these cases was through the recent ingestion of contaminated dairy products.

More importantly, M. bovis was associated with a worse prognosis, including the presence of tuberculomas $(\mathrm{P}=0.032)$, the need for neurosurgery (0.074), and an increased frequency of neurologic sequelae $(\mathrm{P}=0.023)$. The association of a worse prognosis underscores the importance of further classifying the subspecies in all patients with a positive culture for $M$. tuberculosis, especially in patients in which there is no improvement despite treatment. 10 There is no obvious reason to explain the worse prognosis since the recommended treatment is identical for both subspecies, but there is a possibility that these species differ in their mechanisms of the immune response evasion or that the pathogenesis is different.

Another relevant finding was that more than $80 \%$ of the patients had neurological manifestations at onset of the systemic disease. This finding suggests that tuberculosis in the CNS is not a more severe form of pulmonary tuberculosis, but rather a primary phenomenon, or at least with a simultaneous occurrence to the pulmonary disease. It is possible that $M$. tuberculosis organisms with predilection to the CNS comprise different characteristics in pathogenesis or species. ${ }^{12}$

Our conclusions are limited for a number of reasons. First, the retrospective nature of the information is limited to what it is documented in the medical records, and the neurological examinations are usually not rigorous and are subject to variations between examiners. Second, there are no standardized protocols that agree in the optimal time for initiation of medical or surgical treatment, variables that have an enormous impact in the outcome. There were also important differences between the time and number of diagnostic ancillary tests, lumbar puncture PCR, ADA determination, etc. For example, the tuberculin test was not as frequently performed as we expected, possibly due its poor predictive value in an endemic area. Lastly, our Center is a tertiary hospital, which may concede a selection bias in the rates and severity of the disease.

\section{Conclusions}

In our series, the neurologic manifestations of $M$. tuberculosis infection occurred isolated or simultaneous to the onset of the systemic manifestations. CSF Adenosin deaminase determination was a more sensitive method for the early diagnosis than PCR for M. tuberculosis. The isolation of $M$. bovis subspecies was associated with worse prognosis. Initiating empirical treatment with antibiotics for the suspicion of bacterial meningitis was the principal cause of delay for the initiation of anti-tuberculosis treatment and consequently for a poor outcome. Further research is needed to differentiate $M$. tuberculosis with predilection for the CNS from other organisms that do not cause CNS involvement.

\section{References}

1. Ponce-De-Leon A, Garcia-Garcia Md Mde L, Garcia-Sancho MC, et al. Tuberculosis and diabetes in southern mexico. Diabetes Care 2004;27:1584-90.

2. Rock RB, Olin M, Baker CA, Molitor TW,
Peterson PK. Central nervous system tuberculosis: Pathogenesis and clinical aspects. Clin Microbiol Rev 2008;21:24361 , table of contents.

3. Sheu JJ, Yuan RY, Yang CC. Predictors for outcome and treatment delay in patients with tuberculous meningitis. Am J Med Sci 2009;338:134-9.

4. Karandanis D, Shulman JA. Recent survey of infectious meningitis in adults: Review of laboratory findings in bacterial, tuberculous, and aseptic meningitis. South Med J 1976;69:449-47.

5. Misra UK, Kalita J, Roy AK, et al. Role of clinical, radiological, and neurophysiological changes in predicting the outcome of tuberculous meningitis: A multivariable analysis. J Neurol Neurosurg Psychiatr 2000;68:300-3.

6. Saitoh A, Pong A, Waecker NJ,Jr, Leake JA, Nespeca MP, Bradley JS. Prediction of neurologic sequelae in childhood tuberculous meningitis: A review of 20 cases and proposal of a novel scoring system. Pediatr Infect Dis J 2005;24:207-12.

7. Gautam N, Aryal M, Bhatta N, et al. Comparative study of cerebrospinal fluid adenosin deaminase activity in patients with meningitis. Nepal Med Coll J 2007;9:104-6.

8. Rana SV, Chacko F, Lal V, et al. To compare CSF adenosine deaminase levels and CSFPCR for tuberculous meningitis. Clin Neurol Neurosurg 2010;112:424-30.

9. Kato Maeda M, Bobadilla Del Valle M, Martinez Gamboa A, et al. Efficacy and impact of polymerase chain reaction in the diagnosis of extrapulmonary tuberculosis. Rev Invest Clin 2002;54:509-14.

10. Hlavsa MC, Moonan PK, Cowan LS, et al. Human tuberculosis due to mycobacterium bovis in the United States, 1995-2005. Clin Infect Dis 2008;47:168-75.

11. Cicero R, Olivera H, Hernandez-Solis A, et al. Frequency of mycobacterium bovis as an etiologic agent in extrapulmonary tuberculosis in HIV-positive and -negative mexican patients. Eur J Clin Microbiol Infect Dis 2009;28:455-60.

12. Arvanitakis Z, Long RL, Hershfield ES, et al. Tuberculosis molecular variation in CNS infection: evidence for strain-dependent neurovirulence Neurology 1998;50: 1827-32. 